\title{
MODELING FOR POST-YIELD BUCKLING OF REINFORCEMENT
}

\author{
By Rajesh Prasad Dhakal ${ }^{1}$ and Koichi Maekawa ${ }^{2}$
}

\begin{abstract}
Finite element analysis using fiber technique was carried out to study the post-yield buckling mechanism of reinforcing bars. It was found that the softening of compressive stress takes place due to the geometrical nonlinearity associated with the lateral deformation of the compressed bars, especially after the absolute strain exceeds the yielding strain. It was clarified that the post-buckling average stress-strain relationship over the analysis domain can be specified in terms of the product of square root of yield strength and slenderness ratio of the reinforcing bar. Moreover, a unique relationship between the average stress and average strain of reinforcing bars including the effect of buckling is established through various parametric analyses. The comparison of analytical results and proposed model with experimental data showed good agreement.
\end{abstract}

Key words: buckling, lateral deformation, reinforcing bar, average compressive response, point wise stress-strain relationship, geometrical nonlinearity

\section{INTRODUCTION}

In reinforced concrete members, the reinforcing bars might undergo high compressive strain, which induces large lateral deformation of reinforcing bars, hereafter referred to as buckling. Because of geometrical nonlinearity, average compressive stress carried by reinforcing bars decreases in the post-buckling range. However in tension, geometrical nonlinearity does not prevail as no lateral deformation is induced even after yielding. Consequently, the average tensile stress-strain relationship over the specified control volume is exactly same as the point wise tensile stress-strain relationship. Therefore, using similar average stress-strain behavior for reinforcing bars in tension and compression seems unconvincing.

Of course, the point wise stress-strain behavior of reinforcing bars in compression is also the same as that in tension because the point wise relationships are not influenced by the change in geometry (Dodd and RestrepoPosada 1995). Hence, using the point wise stress-strain relationship can account for the lateral deformation of compressed longitudinal reinforcing bars provided that the control volume, over which the average stress-strain relationship is computed, is very small. However, in the finite element structural analysis of larger scale, such small element size is not feasible because of the long computation time and large memory required. For finite element analysis of reinforced concrete structures with substantial element size, an average stress-strain relationship of reinforcing bars that takes into account the effect of buckling is needed. Hence, this research aims to formulate a versatile average stress-strain relationship that can be applied to reinforcing bars with any geometrical and mechanical properties.

To generate the data for model formulation, a parametric study based either on experiment or analysis is necessary. There are some experimental studies performed in the past to clarify the buckling mechanism of bare bar (Monti and Nuti 1992, Rodriguez et al. 1999). Tests are usually conducted within some fixed ranges of material properties, and in some cases we have to consider the boundary conditions, which is hardly reproduced in the tests. For obtaining widely applicable constitutive models, the experiments should consist of test specimens that systematically cover wide range of geometrical as well as mechanical properties. Hence, analytical parametric study is preferred ahead of extensive experimental study, and some available experimental results are used to justify the analytical method and also to verify the proposed constitutive equations.

\section{ANALYTICAL SIMULATION OF BARE BAR BUCKLING}

\section{Fiber Technique for FEM Microanalysis}

A three-dimensional nonlinear finite element analysis program called COM3 [Concrete Model in 3D] (Hauke and Maekawa 1999) is used for the analytical parametric study. Nonlinear space frame elements analyzed by fiber technique (Menegotto and Pinto 1973) are used to model reinforcing bars. In fiber technique, each element is represented using a single line coinciding with the centerline of the member. The member cross section is divided into many cells or sub-elements. The strain of each cell is calculated based on the Euler-Kirchoff's hypothesis, i.e. plane section remains plane after bending. For each fiber strain along the axis of finite element, response is calculated using the material constitutive models representing the local behavior. As is well known, the overall

\footnotetext{
${ }^{1}$ Research Fellow, School of Civil and Structural Engineering, Nanyang Technological University, 50 Nanyang Avenue, Singapore 639798, E-mail: cdhakal@ntu.edu.sg (Formerly: Graduate Student, The University of Tokyo)

${ }^{2}$ Professor, School of Civil Engineering, The University of Tokyo, Hongo 7-3-1, Bunkyo-Ku, Tokyo 113, Japan, E-mail: maekawa@concrete.t.u-tokyo.ac.jp
} 
response of each element is the integrated response of these fibers and the overall response of the member comprises of all the element responses. The implementation of fiber technique is briefly illustrated in Fig. 1.

In this study, isoparametric elements with three-nodes are used. In each node, six degrees of freedom are considered, i.e. the displacements $(u, v$ and $w)$ and rotations $\left(\theta_{x}, \theta_{y}\right.$ and $\left.\theta_{z}\right)$ in three mutually perpendicular directions (local axes $X_{l}, Y_{l}$ and $Z_{l}$ in Fig. 1). Displacement field for an element is calculated from the nodal displacements and the assumed shape functions. Quadratic shape functions are applied for displacement field. Next, strain-displacement relationships are used to compute the generalized strains, which include an axial component $\left(\varepsilon_{0}\right)$, two shear components $\left(\gamma_{z y}, \gamma_{z x}\right)$ and three curvatures $\left(\phi_{x}, \phi_{y}\right.$ and $\left.\phi_{z}\right)$. Based on these generalized strains, the average axial strain of each fiber $\varepsilon_{f}$ is computed. The strain distribution across the cross section is assumed to be linear, which comes from the assumption that plane section remains plane even after bending. In case of bending, the average axial strain of each fiber depends on its distance from the neutral axis. The last two terms (second order derivative terms) in the computation of average axial fiber strain includes the change in geometry due to large displacement of the reinforcing bars during loading. In small strain problems, where the geometry of the structure basically remains unchanged, these higher order terms can be neglected. However, large lateral displacement of reinforcing bars cannot be captured without giving due consideration to geometrical nonlinearity. This fact is analytically proved afterwards. Based on this axial strain, fiber axial stress is calculated from the point wise stress-strain relationship. These fiber stresses across the cross section are integrated to obtain the stress carried by the element.

\section{Effect of Buckling in Average Behavior}

Following the aforementioned computation scheme, overall average response would become similar to the local response if only one element is used to model the bare bar length. To capture the associated geometrical nonlinearity, large number of finer elements should be used. A schematic diagram explaining the effect of reinforcement buckling on the average response is depicted in Fig. 2. Here, the length between two clamped boundaries $L$ is defined as referential length, over which the average stress-strain relationship is to be generated. Before the lateral deformation is induced, the stress and strain distribution along the length as well as across the cross-section is uniform. Therefore, overall average behavior is the same as local behavior. However, once the reinforcement deforms laterally under high compression, the stress and strain distributions along the length and across the bar section change significantly. As shown in Fig. 2, laterally deformed part of the referential length experiences compression hardening, and the rest is subjected to unloading. Moreover, curvature exists due to the lateral deformation, and the strain across the bar section is not constant but varies linearly. Obviously, the value of curvature depends on the position of the concerned section along the length. For example, at mid-height where maximum lateral deformation is experienced, the inner fibers are in compression hardening state whereas the outer fibers are in unloading state. Because the unloading stiffness is significantly higher compared to the hardening stiffness, a small decrease in fiber strain causes substantial decrease in the fiber stress compared to the increase in fiber stress due to an equivalent increase in fiber strain. Consequently, the average stress of the overall referential length becomes smaller than the local response.

\section{Preliminary Microanalysis}

As shown in Fig. 3, the reinforcing bar is modeled as a vertical column of length $L$ and diameter $D$. The total length $L$ is discretized into several small elements. Monotonic downward displacement $\Delta$ is applied at the top of the reinforcement. To know the effect of end condition, a pair of analyses is carried out with considering fixed ends and hinged ends, respectively. An elastic-perfectly plastic stress-strain relationship without hardening is used as the local material model, and the local yield stress and Young modulus of steel are assumed to be $400 \mathrm{MPa}$ and $200 \mathrm{GPa}$, respectively. The average load-displacement relationships computed with these two boundary conditions are compared in Fig. 3. It is found that allowing rotations at the extreme ends causes severe softening of the average response. In order to simulate the bare bar compression tests (Mander et al. 1984; Monti and Nuti 1992), the two ends of the reinforcing bars are represented by fixed nodes, which coincides with the experimental loading condition; i.e. both displacement and rotation are not allowed to take place at these nodes.

As mentioned earlier, the lateral deformation of reinforcing bars can be predicted only if the geometrical nonlinearity is given due consideration in the analysis. In order to verify this statement, a pair of analyses with and without considering geometrical nonlinearity is performed, and the average response are compared in Fig. 4(a). If geometrical nonlinearity is not considered; i.e. the second order terms are neglected in the computation of fiber strain, the post-yield buckling phenomenon cannot be predicted, and the average response becomes the same as the local response. Next, mesh sensitivity analysis is conducted to obtain the influence of mesh discretization in average response. A reinforcing bar with $L=16 \mathrm{~cm}, D=16 \mathrm{~mm}, f_{y}=400 \mathrm{MPa}$ is analyzed with varying number of elements. 
The average load-displacement curves are compared in Fig. 4(b). Using only one element rendered the average bar response exactly follow the assumed local behavior, and could not predict the softening of the average compression in spite of considering geometrical nonlinearity. As expected, the average responses obtained using finer elements exhibit increased softening, and the mesh dependency nearly vanishes for more than 4 elements. Hence, at least four or more elements are used for further analyses.

Next, the lateral deformation profile and longitudinal as well as transverse distribution of fiber strain and stress are explained. Three instants denoted by points $A, B$ and $C$ in Fig. 4(b) are selected for comparison. Point $A$ refers to the instant just before the average stress starts decreasing, and points $B$ and $C$ lie in the softening phase. The lateral displacement profiles plotted in Fig. 4(c) indicate that the bar deforms laterally only after point $A$, and this lateral deformation is maximum at the mid-height. Similarly, the average strain (strain at the center of the cross-section) profiles plotted in Fig. 4(d) reveal that the bar experiences uniform compression until point $A$, i.e. the starting point of buckling. This uniformity is lost afterwards and some portions, especially near the fixed support and around the mid-height, experience continuous increase in compressive strain while strains in other parts decrease.

In Fig. 5, the fiber strain and stress distributions across the bar section at the mid-height during phases $\mathrm{B}$ and $\mathrm{C}$ are plotted. The bar cross-section lies in the center of the plotted rectangular domain in the figure, and the corners that are extrapolated from the data inside the bar section should be disregarded. Both the strain and stress are constant throughout the bar section at phase $A$, and hence are not shown in the figure. However, curvature is induced afterwards due to the lateral deformation. Consequently, the strain linearly changes across the bar section, and the stress distribution becomes highly irregular due to different post-yield loading and unloading stiffness. Due to the elastic-perfectly plastic material model used in the analysis, the compressive stress in the fibers does not exceed yield stress in spite of large fiber strain. In contrast, the compressive stress in the unloading fibers is less than the yield stress, and some extreme fibers even experience tensile stress. As claimed earlier, this complex mechanism leads to the softening of average compressive stress with increase in lateral deformation.

\section{General Discussion on FEM Microanalysis}

In the mesh for microanalysis, all nodes of all elements were perfectly aligned along the same vertical line and no initial imperfection was provided to induce lateral deformation. In spite of this fact, the analytical solution could capture the buckling mechanism. The two possible modes of deformation of a bar under axial compression are axial mode representing longitudinal shortening and bending mode representing the usual buckling shape. The axial mode always leads to local stress-strain behavior whereas the bending mode triggers the softening of average stress provided that the material nonlinearity and path-dependency are considered in the constitutive model. Both of these modes satisfy the requirements; i.e. the compatibility, equilibrium and the constitutive equations. However, the energy associated with the bending mode is smaller than that with axial mode. Hence, if geometrical nonlinearity is considered and the intermediate nodes are free to deform laterally, the finite element solution selects the bending mode, as it requires less energy during the nonlinear iteration process.

For further understanding, the analysis of a bar under compression was performed with geometrical nonlinearity but without any path-dependency; i.e. stress reversal during cyclic loading was not considered. In other words, once the compressive strain exceeds yielding strain, the stress is kept constant at yield stress regardless of the strain path (unloading or reloading). As shown in Fig. 6, the average stress-strain curve in this case is found to be the same as local stress-strain relationship in spite of the presence of large lateral deformation. Although high curvature (strain gradient) across the section could be observed, the stresses at all fibers were equal because of the path-independent constitutive model. Consequently, in spite of the geometrical nonlinearity, softening of the average stress could not be observed. This can also explain why the average response is nearly the same as local response until yielding. For reinforcing bars with normal slenderness ratio, lateral deformation does not start in the elastic range and the bar deforms axially rendering the average behavior to be similar to the local behavior. Nevertheless in very slender bars, lateral deformation might take place before yielding. The loading and unloading stiffness in elastic range are both equal to the Young's modulus and a small strain gradient due to the lateral deformation does not cause significant difference between the local and the average stress. However, when the average strain is close to yield strain, even a small strain gradient might be influential as some fibers experience hardening and the others undergo elastic unloading, causing average stress to be slightly less than the local stress.

Euler's theorem is mostly used to calculate the buckling load of axially compressed longitudinal bars. However, it is based on the assumption that the bar behaves elastically throughout the loading. Hence for short bars, the buckling load predicted by Euler's formula is usually higher than the yield load. But, because of the elasto-plastic nature of the reinforcing bars, the load cannot linearly increase in the post-yield range, and Euler's theory is no longer applicable. In this context, the FEM microanalysis predicts that the buckling starts in the plastic region, where 
the axial stress is slightly higher than or equal to yield stress but the strain is substantially higher than the yielding strain. For normal strength steel bars $\left(f_{y} \sim 400 \mathrm{MPa}\right)$ with slenderness ratio less than or equal to 35 and fixed at both ends, Euler's formula predicts the critical buckling load to be more than yielding load. Keeping in mind that the tie spacing in RC members almost never exceeds 35 times longitudinal bar diameter, it can be said that Euler's theory cannot be directly used, and FEM simulation serves as a more reliable method. Needless to mention, for bars with higher slenderness ratio, buckling starts in elastic range and Euler's formula gives accurate prediction of buckling load that is smaller than the yield load.

The softening of average stress in post-buckling range is due mainly to the difference between the stiffness for post-yield loading and unloading paths. The average response depends on both geometrical nonlinearity and the path-dependency, and the influence of path-dependency becomes crucial only when the geometrical nonlinearity is taken into account. On the other hand, the lateral deformation can be simulated only with geometrical nonlinearity but the softening of the post-buckling average stress cannot be captured without considering the path-dependency in the nonlinear material model. The average compressive stress-strain relationships predicted analytically with assumed material model might not be exact but is certainly not far from the correct one. For sure, if unloading and hardening properties are correctly known in advance, the correct average constitutive model of reinforcing bars with any type of point wise stress-strain relationship can thus be obtained analytically.

\section{VERIFICATION AND PARAMETRIC STUDY}

\section{Verification of Microanalysis}

For verification of analytical method, the computed results are compared with experimental ones (Mander et al. 1984). The experiment consists of direct compression tests of five short medium strength $\left(f_{y}=290 \mathrm{MPa}\right)$ reinforcing bars with different slenderness ratios $(5.5,6,6.5,10$ and 15). The schematic test setup, point wise stress-strain relationship and comparative normalized average compressive stress-strain curves are shown in Fig. 7. Note that the hardening material model obtained through sample bar test was used in the verification analysis. The experimental and analytical curves are compared for only three different slenderness ratios, as the results of the first three cases are found to be very close to each-other in experiment as well as analysis, and only one representative case $(L / D=6)$ among these three was chosen. The results of FEM microanalysis are in good agreement with the experimental curves giving ample proof of the reliability of the analysis.

Next, the monotonic tests of Monti and Nuti (1992) are adopted for the verification of monotonic behavior. The experiment consists of series of monotonic tests on steel reinforcing bars with different slenderness ratios $(5,8$ and 11) and three different bar diameters of $16 \mathrm{~mm}, 20 \mathrm{~mm}$ and $24 \mathrm{~mm}$ were used for each slenderness ratio. Here, the average of the three responses (with different diameters) for each slenderness ratio is used for comparison with FEM microanalysis. As the details of the material properties were not mentioned, the point wise stress-strain relationship was fairly assumed to match the average response of the bar with slenderness ratio 5 , because it was reported that the behavior of this shortest bar almost coincides with the material model. The point wise stress-strain relationship used and the normalized average stress-strain curves are shown in Fig. 8. It can be observed that the results of FEM microanalysis are in fair agreement with the experimental results for all three cases of monotonic loading. However, small difference can be observed in the early hardening range of the bar with slenderness ratio 5 . This is due to the fact that the nonlinear hardening of the bar with slenderness ratio 5 was represented by using an average linear hardening range in the point wise stress-strain relationship.

These verifications give ample evidence that FEM microanalysis with coupled geometrical and material nonlinearity and path-dependent material models can reliably predict the buckling mechanism and also the average stress-strain behavior of axially compressed reinforcing bars. In addition, the accuracy is found to be good enough for engineering purposes. Hereafter, detailed parametric study is conducted using FEM analysis to obtain unified and versatile equations that can represent the average compressive stress-strain relationship of reinforcing bars.

\section{Effect of Length and Diameter}

It is well known that the average behavior of reinforcing bar is very sensitive to its length and diameter. To elaborate more in this regard, two sets of parametric analyses are conducted with constant diameter and different lengths as well as constant length and different diameters. The normalized average responses are compared in Fig. 9(a) and (b). The softening of average stress becomes steeper with the increase in reinforcement length provided that the bar diameter is constant. Similarly, if the reinforcement length is kept constant, the softening becomes milder with increase in bar diameter. Both of these give similar qualitative relationship; i.e. the more slender the bar is, more 
severe is the softening of average stress. In order to obtain quantitative interrelationship, two pairs of cases, so that the slenderness ratio (length to diameter ratio) is the same but the length and diameter are different, are compared in Fig. 9(c). It is found that irrespective of the separate values of length and diameter, the normalized average response is similar for the same slenderness ratio. Finally, the normalized average responses for different slenderness ratios, assuming elastic-perfectly plastic behavior and $f_{y}=400 \mathrm{MPa}$, are shown in Fig. 9(d). Agreeing with the conclusions of previous studies (Dhakal and Maekawa 2000; Mau and El-Mabsout 1989; Monti and Nuti 1992), the results showed that buckling is delayed and the post-buckling degradation of average response decreases with the decrease in slenderness ratio of reinforcing bars.

\section{Effect of Yield Strength}

The authors believe that the strength of reinforcing bar also influences the average behavior in compression. To explore the interrelationship, two sets of sensitivity analyses are performed with constant slenderness ratio and different yield strengths. The comparative normalized average stress-strain curves for slenderness ratios 5 and 10 and yield strengths ranging from $100 \mathrm{MPa}$ to $1600 \mathrm{MPa}$ are presented in Fig. 10(a) and (b) respectively. The softening of average stress becomes steeper with the increase in yield strength provided that the slenderness ratio is constant. Agreeing with previous study (Mau and El-Mabsout 1989), the average response of high strength bar is found to become more brittle than that of lower strength bar. It was reported that the average response becomes nearly the same as the local response if $L / D$ ratio is equal to or smaller than 5 (Mau 1990; Monti and Nuti 1992). However, the microanalysis suggested that this is not the reality and this critical slenderness ratio depends on the yield strength of the reinforcing bar. For example, the average response of bar with slenderness ratio 5 is very close to local response for $f_{y}=100 \mathrm{MPa}$ but the difference between the local and average responses is very big for $f_{y}=1600 \mathrm{MPa}$. Hence, rather than specifying the relationship in terms of slenderness ratio and yield strength separately, a combined term including both of these parameters seems more suitable.

For this purpose, two pairs of special cases are compared with each other in Fig. 10(c). The first pair includes two cases, one with $f_{y}=100 \mathrm{MPa} ; L / D=10$ and the other with $f_{y}=400 \mathrm{MPa} ; L / D=5$. Similarly, the second pair includes the cases with $f_{y}=400 \mathrm{MPa} ; L / D=10$ and with $f_{y}=1600 \mathrm{MPa} ; L / D=5$. Interestingly, the normalized average responses of the two cases in each pair were exactly similar to each other, suggesting that there is a unique interrelationship between the parameter $L / D \sqrt{ } f_{y}$ and the average response. Although some studies on modeling of average compressive response of reinforcing bars have been performed in the past (Mau and El-Mabsout 1989; Mau 1990; Monti and Nuti 1992), this unique interrelationship between yield strength and average compressive response was not realized. Next, the normalized average responses for different values of $L / D \sqrt{ } f_{y}$, using elastic-perfectly plastic point wise stress-strain relationship, are shown in Fig. 10(d). As expected, buckling happens earlier and the post-buckling average stress degrades faster with increase in the value of this parameter.

\section{Reinforcing Bars with Strain Hardening}

The earlier discussions were based on the microanalysis of reinforcing bars of elastic-perfectly plastic material. In contrast, commonly used reinforcing bars exhibit hardening after a yield plateau in the post-yield range, though the hardening stiffness and range may vary between the bars produced in different countries. To cover all types of reinforcing bars, the parametric study hereafter is conducted for linear hardening material, which along with elasticperfectly plastic material constitute the two opposite extremes of all possible hardening mechanisms. The assumed point wise stress-strain relationship is shown in Fig. 11(a), and the average compressive stress-strain curves of bars with slenderness ratio varying between 5 and 15 are shown in Fig. 11(b)-(d) for yield strength equal to 200, 400 and $800 \mathrm{Mpa}$, respectively. As expected, the softening of average post-buckling compressive stress for the same slenderness ratio becomes steeper with the increase in yield strength. Moreover, the starting point of negative slope is delayed due to strain hardening effect, and the absolute value of normalized stress is also higher than that in elasticperfectly plastic reinforcement. The FEM microanalysis could successfully capture the average response of high strength bars $\left(\mathrm{f}_{\mathrm{y}}=800 \mathrm{Mpa}\right)$ that is distinctly more brittle compared to the average response of normal strength bars with same slenderness ratio.

Note that the negative slope of the average stress-strain curves in the later descending stage is nearly constant (approximately equal to $2 \%$ of Young's modulus), irrespective of the value of the parameter $L / D V f_{y}$ as in the elastoplastic bars. Similarly, the average compressive stress nearly becomes constant after it decreases to $20 \%$ of the yield strength. This information can greatly assist in the modeling of average compressive behavior of reinforcing bars. In this case too, the average response was found to uniquely depend on the parameter $L / D \sqrt{ } f_{y}$, irrespective of separate values of $L, D$ and $f_{y}$. As the response of these two extreme cases (elastic-perfectly plastic and linear hardening) 
indicate similar interrelationships between the slenderness ratio, yield strength and the average compressive response, the same should be applicable to reinforcing bars with hardening mechanisms in between these extremes. Hence, the limiting $L / D$ ratio below which the average behavior is close to the material model can be better approximated by $\left(100 / \mathfrak{V}_{y}\right)$ rather than 5 regardless of bar strength as recommended in previous studies.

\section{MODELING OF AVERAGE STRESS-STRAIN RELATIONSHIP}

\section{Average Model for Monotonic Compression}

As proved earlier, the average behavior of reinforcement in compression can be reliably captured if sufficiently small elements are used in the finite element analysis. Nevertheless, it is impractical and tedious to use such small elements in the analysis of real size reinforced concrete structures. While using elements of substantial size, the point wise material models should be replaced with the average stress-strain relationships defined over the specified finite element. Here, an average stress-strain relationship for reinforcing bars including the effect of buckling is formulated.

Through the analytical parametric study, various facts regarding the average behavior of reinforcing bars in compression are revealed. Some of them are: 1) The average compressive stress-strain relationship solely depends on the product of slenderness ratio and yield strength. 2) The average compressive stress becomes smaller than the local stress after the initiation of buckling, and the starting point of average stress degradation also depends on the point wise stress-strain relationship. 3) The stress degradation rate in the later stage of post-buckling average stress-strain curve is nearly constant with a negative slope approximately equal to $2 \%$ of the Young's modulus. 4) The average compressive stress becomes constant after it reaches the value equal to $20 \%$ of the yield strength.

Guided by these unique interrelationships, an average monotonic compressive stress-strain model is proposed, the general layout of which is sketched in Fig. 12. An intermediate point $\left(\varepsilon^{*}, \sigma^{*}\right)$ is established, after which a constant negative stiffness equal to $0.02 E_{s}$ is assumed until the average stress becomes equal to $0.2 f_{y}$. Here, $E_{s}$ and $f_{y}$ are Young's modulus and yield strength, respectively. To represent the aforementioned mechanisms, following equations relating the absolute average compressive stress with the absolute average compressive strain of reinforcing bar are proposed.

$$
\begin{aligned}
& \frac{\sigma}{\sigma_{l}}=1-\left(1-\frac{\sigma^{*}}{\sigma_{l}^{*}}\right)\left(\frac{\varepsilon-\varepsilon_{y}}{\varepsilon^{*}-\varepsilon_{y}}\right) ; \quad \text { for } \varepsilon_{y}<\varepsilon \leq \varepsilon^{*} \\
& \sigma=\sigma^{*}-0.02 E_{S}\left(\varepsilon-\varepsilon^{*}\right) ; \quad \sigma \geq 0.2 f_{y} ; \quad \text { for } \varepsilon>\varepsilon^{*}
\end{aligned}
$$

Here, $\sigma_{l}$ and $\sigma_{l}{ }^{*}$ are the local responses corresponding to $\varepsilon$ (current strain) and $\varepsilon^{*}$ (strain at intermediate point), respectively. Similarly, $\varepsilon_{\mathrm{y}}$ and $E_{s}$ are yielding strain and Young's modulus of the reinforcement. To make the model applicable to bars with all types of material model, the stresses at and before the intermediate point are normalized with respect to the stresses computed from the point wise stress-strain relationship at the corresponding strain value. This normalization technique also allows the shape of the average response before this intermediate point to look like point wise stress-strain curve, which was distinctly observed in all microanalysis results. The coordinates of the intermediate point $\left(\varepsilon^{*}, \sigma^{*}\right)$ can be calculated by means of following equations.

$$
\begin{array}{ll}
\frac{\varepsilon^{*}}{\varepsilon_{y}}=55-2.3 \sqrt{\frac{f_{y}}{100}} \frac{L}{D} ; & \varepsilon^{*} / \varepsilon_{y} \geq 7 \\
\frac{\sigma^{*}}{\sigma_{l}^{*}}=\alpha\left(1.1-0.016 \sqrt{\frac{f_{y}}{100}} \frac{L}{D}\right) ; & \sigma^{*} \geq 0.2 f_{y}
\end{array}
$$

Note that equations (2) and (3) are derived from the microanalysis results of bars with linear hardening behavior plotted in Fig. 13. When checked with the results of elastic-perfectly plastic bars, it was found that the normalized strain at the intermediate point $\varepsilon^{*}$ was not much different, and equation (2) was still applicable without any amendment. Nevertheless, the normalized value of stress at the intermediate point $\sigma^{*}$ was smaller than that in linear 
hardening bars. Hence, for bars with more realistic hardening model, $\varepsilon^{*} / \varepsilon_{y}$ is expected to be unaffected but $\sigma^{*} / \sigma_{l}$ is expected to be smaller than in linear hardening bars and larger than in elasto-plastic bars. To account for this change, a coefficient $\alpha$ is included in the formulation of $\sigma^{*}$. For the two extreme cases assumed earlier for the parametric study, the value of $\alpha$ is found to be 1.0 for linear hardening bars, and 0.75 for perfectly elasto-plastic bars. For bars with limited hardening range, in which most of the industrial products fall, it should be chosen between 0.75 and 1 . If the hardening stiffness is very small and the hardening range in terms of strain is short, the value should be closer to 0.75. On the other hand, if the hardening stiffness is significant and lasts for large strain range, the value should be closer to 1.0 .

\section{Giuffre-Menegotto-Pinto Model for Cyclic Loops}

As the framework for unloading and reloading behavior during strain reversals, the authors adopt GiuffreMenegotto-Pinto model (CEB 1996). As illustrated in Fig. 14, it uses a smooth transition curve asymptotic to the tangents at the point of stress reversal and the point of maximum/minimum strain in the loading history. The original model of Giuffre-Menegotto-Pinto takes on the form of normalized stress-strain relationship, as expressed in (4)-(6).

$$
\begin{aligned}
& \sigma_{e q}=b \varepsilon_{e q}+\frac{(1-b) \varepsilon_{e q}}{\left(1+\varepsilon_{e q}^{R}\right)^{1 / R}} \\
& \varepsilon_{e q}=\frac{\varepsilon-\varepsilon_{r}}{\varepsilon_{0}-\varepsilon_{r}} ; \sigma_{e q}=\frac{\sigma-\sigma_{r}}{\sigma_{0}-\sigma_{r}} \\
& R=R_{0}-\frac{a_{1} \xi}{a_{2}+\xi}
\end{aligned}
$$

These equations represent a smooth transition from the unloading/reloading stiffness at the starting point to the hardening stiffness at the minimum/maximum strain point in the loading history. In (4), $\varepsilon_{e q}$ and $\sigma_{e q}$ are normalized strain and stress parameters and can be calculated according to (5), where $\left(\varepsilon_{0}, \sigma_{0}\right)$ is the intersection point of the two asymptotes (point $A$ in Fig. 14), and $\left(\varepsilon_{r}, \sigma_{r}\right)$ is the unloading/reloading point (point $D$ in Fig. 14). These points are updated after each strain reversal. Similarly, $R$ is a parameter that influences the shape of the transition curve, and is expressed as shown in (6). Here, $\xi$ is the normalized strain difference between the current asymptote intersection point (point $A$ in Fig. 14) and the maximum/minimum strain ever experienced in the past loading history (point $B$ in Fig. 14). $R_{0}$ is the value of the parameter $R$ during first loading and should be experimentally defined along with the constants, $a_{1}$ and $a_{2}$. The larger the positive value of $R$ is, more abrupt the change of stiffness of the loop around the intersection points of the two tangents becomes. Provided that the resulting value of $R$ is positive, the transition becomes smoother if the value of $a_{1}$ increases or that of $R_{0}$ and $a_{2}$ decreases. However, making the transition smoother will increase the difference between the maximum/minimum stress achieved earlier and the stress represented by the transition curve at the target point. Hence, one should be careful while selecting the values of these constants so that the value of $R$ remains positive and the stress difference at the target point remains small to avoid sudden stress jump that might cause convergence problem during FEM iterations. As these constants do not influence the monotonic curve and small changes in their values marginally affect the transition shapes during strain reversals, the authors have adopted $R_{0}=20 ; a_{1}=18.5$; and $a_{2}=0.15$ after extensive checking.

In (4), $b$ is the strain-hardening ratio, defined by the ratio between slope at the minimum/maximum point in the loading history $\left(E_{l}\right.$ in Fig. 14) and slope at the unloading/reloading point. The unloading stiffness from tension is equal to $E_{s}$; i.e. the Young's modulus of reinforcement. However, it has been observed that reloading stiffness from post-buckling compression state is significantly smaller than $E_{s}$ (Suda et al. 1996; Monti and Nuti 1992). Hence, the reloading stiffness from post-buckling compression state is reduced to $E^{*}$ as expressed in (7).

$$
E^{*}=E_{S}\left(\frac{\sigma_{\min }}{\sigma_{\max }}\right)^{2} \quad E_{s} \geq E^{*}
$$




\section{Verification of Proposed Computational Model}

A complete path-dependent cyclic model relating the average stress with average strain is formulated by combining the proposed monotonic compressive average stress-strain relationship with tension envelope of the bare bar and Giuffre-Menegotto-Pinto model for the unloading/reloading loops. For verification, thus generated cyclic model is compared with the results of FEM microanalysis as well as some experimental results.

First, the performance of the proposed model is compared with FEM microanalysis and experimental results of the monotonic tests (Monti and Nuti 1992) in Fig. 15(a). As the point wise cyclic stress-strain relationship of the bar material was not reported, the FEM microanalysis could not be carried out for cyclic tests, and the average cyclic responses observed in the experiment are compared only with those computed by the proposed model, as illustrated in Fig. 15(b)-15(d) for slenderness ratios 5, 8 and 11, respectively. As the hardening stiffness in the material model is significant $\left(0.055 E_{s}\right)$ but lasts only for $8 \varepsilon_{y}$, the coefficient in the proposed model is assumed as $\alpha=0.9$ according to the earlier recommendation. As seen in the figure, the monotonic curves predicted by the model for different slenderness ratios are close to the FEM microanalysis and experimental results. Moreover, it can also be observed that the proposed model is in good agreement with the experimental results for all three cases of cyclic loading, giving ample evidence that the proposed monotonic average compressive stress-strain relationship combined with Giuffre-Menegotto-Pinto cyclic model can be reliably used.

Nevertheless, the verification for cyclic loading is conducted within the range of 3\% tensile and compressive strains; i.e. around 15 times yielding strain for normal strength bars. Although the $3 \%$ compressive strain is difficult to be reached in the reinforcing bars in actual RC structures, tensile strain induced in reinforcing bars in RC structures during strong earthquakes might be larger than the verified range. Test data measuring much higher strain are scarce, which might be due to the difficulty in measuring higher strain with the commonly used strain gauges. Note that the average compressive stress of the most slender bar $(\mathrm{L} / \mathrm{D}=11)$ unloaded from large tensile strain starts to decrease before reaching the compressive strain zone because of the negative slope at the target point. This behavior, observed both in experiment and model prediction, is qualitatively supporting the outcome of previous study (Rodriguez et al. 1999) that the compressive stress degradation point (defined as buckling onset point) depends on the maximum tensile strain reached before a strain reversal. The effect of maximum tensile strain might be more pronounced if the strain reversal is from much larger strain, and the proposed model needs to be verified for such large strain cyclic tests before drawing quantitative conclusions.

Next, the proposed model is compared with FEM microanalysis for some general cases. Figs. 16 and 17 show the comparison between proposed model and FEM analysis for reinforcing bars with linear hardening and elasticperfectly plastic point wise stress-strain relationships, respectively. The comparisons are performed for different values of the parameter $F L D^{*}=L / D \downarrow\left(f_{y} / 100\right)$. As established earlier, the coefficient $\alpha$ in the proposed model is assumed as unity for the linear strain hardening case and 0.75 in elastic-perfectly plastic case. Average responses predicted by the proposed model are very close to those by FEM microanalysis for almost all cases. As the model is valid for these two extreme cases, the authors believe that it can represent the behavior of bars with any hardening range. The earlier verification of the models with the available test data of bars with reasonable hardening range also advocates this claim.

\section{CONCLUSIONS}

Microanalyses based on fiber technique using finite elements with small size were conducted to predict the average behavior of reinforcing bars in compression accompanying geometrically large nonlinearity. It was revealed that the average compressive behavior depends only on one parameter; i.e. $L / D V f_{y}$ (product of slenderness ratio and square root of yield strength). It was also found that the conclusion from some previous studies, stating that the average compressive response of reinforcing bars with slenderness ratio equal to or less than 5 is the same as the local response, is not always true, especially in case of high-strength bars. Based on the results of parametric study, an average compressive stress-strain model is proposed that can be applied for reinforcing bars with any geometrical as well as mechanical properties. Giuffre-Menegotto-Pinto model for cyclic loops is combined with the proposed model to yield a complete path-dependent cyclic model. The comparison of the microanalysis as well as the proposed model with some experimental results showed good agreement and verified the reliability of proposed model for both monotonic and cyclic loading. 


\section{ACKNOWLEDGEMENTS}

The authors gratefully acknowledge TEPCO Research Foundation and Grant-in-aid for scientific research No. 11355021 for providing financial support to accomplish this research.

\section{APPENDIX. REFERENCES}

CEB (1996). RC Elements under Cyclic Loading - State of the Art Report, Thomas Telford.

Dhakal, R. P., and Maekawa, K. (2000). "Post-peak cyclic behavior and ductility of reinforced concrete columns," Modeling of Inelastic Behavior of RC Structures under Seismic Loads, ASCE, 193-216.

Dodd, L. L. and Restrepo-Posada, J. I. (1995). "Model for predicting cyclic behavior of reinforcing steel." Journal of Structural Engineering, 121 (3), 433-445.

Hauke, B., and Maekawa, K. (1999). "Three-dimensional modeling of reinforced concrete with multi-directional cracking." Journal of Materials, Concrete Structures and Pavements, JSCE, 634 (45), 349-368.

Mander, J. B., Priestley, M. J. N., and Park, R. (1984). "Seismic design of bridge piers." Research Report 84-2, Department of Civil Engineering, University of Canterbury, New Zealand.

Mau, S. T. (1990). "Effect of tie spacing on inelastic buckling of reinforcing bars." ACI Structural Journal, 87 (6), $671-678$.

Mau, S. T., and El-Mabsout, M. (1989). "Inelastic buckling of reinforcing bars." Journal of Engineering Mechanics, 115 (1), $1-$ 17.

Menegotto, M., and Pinto, P. E. (1973). "Method of analysis of cyclically loaded RC plane frames including changes in geometry and non-elastic behavior of elements under normal force and bending." Preliminary Report IABSE, 13, 15-22.

Monti, G., and Nuti, C. (1992). "Nonlinear cyclic behavior of reinforcing bars including buckling." Journal of Structural Engineering, 118 (12), 3268-3284.

Rodriguez, M. E., Botero, J. C., and Villa, J. (1999). "Cyclic stress-strain behavior of reinforcing steel including effect of buckling." Journal of Structural Engineering, 125 (6), 605-612.

Suda, K., Murayama, Y., Ichinomiya, T., and Shimbo, H. (1996). "Buckling behavior of longitudinal reinforcing bars in concrete column subjected to reverse lateral loading." Proc., $11^{\text {th }}$ World Conference on Earthquake Engineering, Acapulco, Mexico, CD ROM Paper No. 1753.

\section{APPENDIX. NOTATION}

$a_{1}, a_{2}=$ experimental constants to represent cyclic loop;

$b=$ strain-hardening factor; $b=E_{l} / E_{s}$ or $b=E_{l} / E^{*}$;

$D=$ diameter of reinforcing bar;

$E_{s}=$ Young's modulus of reinforcing bar;

$E_{l}=$ tangential stiffness at maximum/minimum strain;

$E_{s h l}, E_{s h 2}=$ first and second stage hardening stiffness;

$E^{*}=$ modified reloading stiffness from minimum strain;

$f_{y}=$ yield strength of reinforcing bar;

$F L D^{*}=$ parameter, $F L D^{*}=L / D \downarrow\left(f_{y} / 100\right)$;

$L=$ test length of reinforcing bar;

$R=$ parameter influencing the shape of the transition curve;

$R_{0}=$ value of $R$ during first loading;

$u, v, w=$ displacements along $X, Y$ and $Z$ axes;

$x, y=$ distance to a fiber along $X$ and $Y$ axes from center;

$\alpha=$ coefficient depending on the local behavior of bar;

$\varepsilon, \sigma=$ average strain and average stress;

$\varepsilon^{*}, \sigma^{*}=$ strain and stress at the intermediate point;

$\varepsilon_{f}=$ axial strain of a reinforcement fiber;

$\varepsilon_{0}, \sigma_{0}=$ strain and stress at the intersection of two tangents;

$\varepsilon_{r}, \sigma_{r}=$ maximum/minimum strain and corresponding stress;

$\varepsilon_{s h 1}, \varepsilon_{s h 2}=$ first and second stage local hardening strain;

$\varepsilon_{y}=$ yielding strain of the longitudinal reinforcement;

$\varepsilon_{e q}, \sigma_{e q}=$ normalized strain and stress,

$\varepsilon_{e q}=\left(\varepsilon-\varepsilon_{r}\right) /\left(\varepsilon_{0}-\varepsilon_{r}\right) ; \sigma_{e q}=\left(\sigma-\sigma_{r}\right) /\left(\sigma_{0}-\sigma_{r}\right) ;$

$\phi_{x}, \phi_{y}, \phi_{z}=$ curvatures with respect to $X, Y$ and $Z$ axes;

$\gamma_{z y}, \gamma_{z x}=$ in-plane and out-of-plane shear strains;

$\theta_{x}, \theta_{y}, \theta_{z}=$ rotations with respect to $X, Y$ and $Z$ axes;

$\sigma_{\min }, \sigma_{\max }=$ stresses at the minimum and maximum strain;

$\sigma_{l}, \sigma_{l}{ }^{*}=$ local stresses at current strain and $\varepsilon^{*}$, respectively;

$\xi=$ normalized strain difference between the intersection point of two asymptotes and the maximum/minimum strain. 


\section{LIST OF FIGURES:}

FIG. 1. Fiber technique adopted in the finite element analysis

FIG. 2. Effect of reinforcement buckling in average response

FIG. 3. Simulation of bare bar compression test and effect of end condition

FIG. 4. a) Effect of geometrical nonlinearity

b) Effect of mesh dicretization

c) Lateral displacement profile after buckling

d) Variation of average strain along the length

FIG. 5. Strain and stress distributions across the cross-section after buckling

FIG. 6. Effect of path-dependency of fiber constitutive model on the average behavior

FIG. 7. Comparison with experimental results (Mander et al. 1984)

FIG. 8. Comparison with experimental results (Monti and Nuti 1992)

FIG. 9. Effect of length and diameter on the average response

FIG. 10. Effect of yield strength on the average response

FIG. 11. Average compressive responses of reinforcements with strain hardening

FIG. 12. Schematic representation of proposed model

FIG. 13. Determination of intermediate point based on microanalysis

FIG. 14. Giuffre-Menegotto-Pinto model for cyclic loop

FIG. 15. Comparison with monotonic and cyclic test results (Monti and Nuti 1992)

FIG. 16. Comparison of model with FEM analysis (linear strain-hardening case)

FIG. 17. Comparison of model with FEM analysis (elastic-perfectly plastic case) 


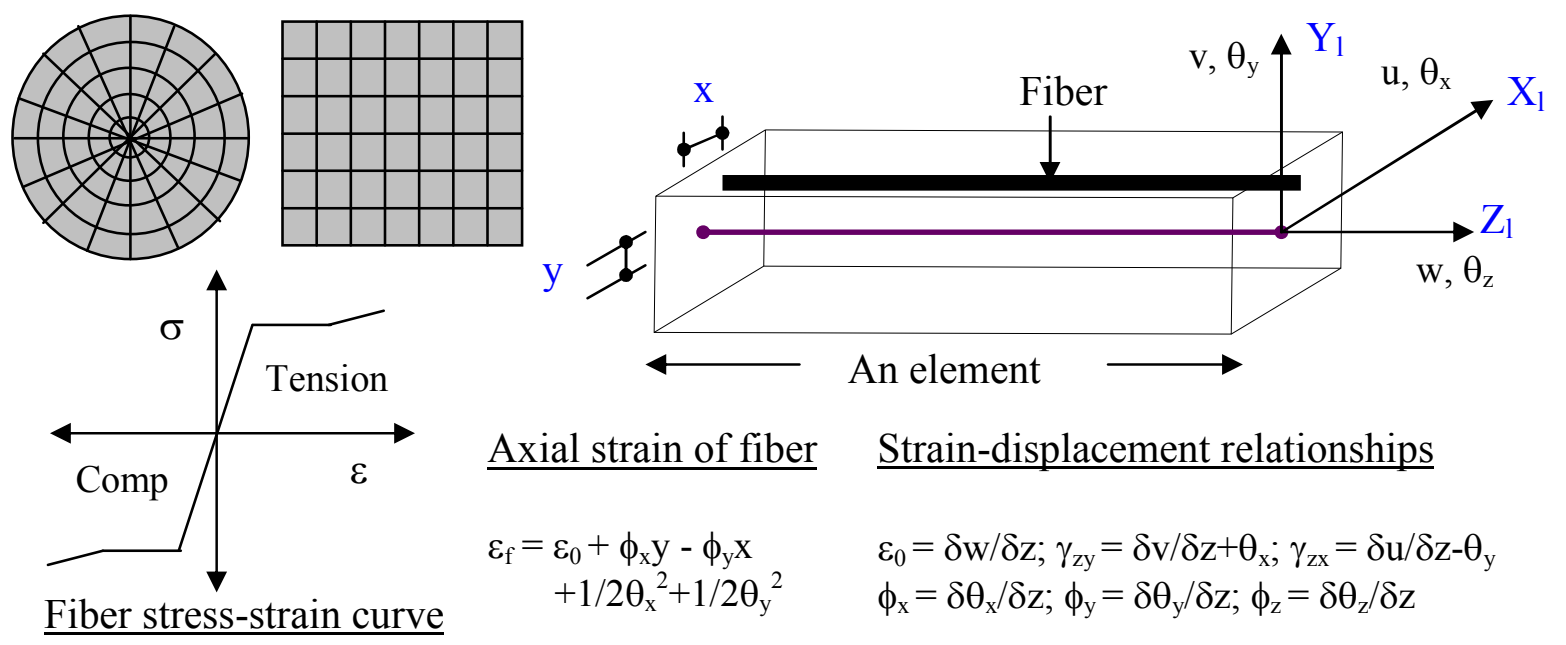

Fig. 1 


\section{Before buckling}

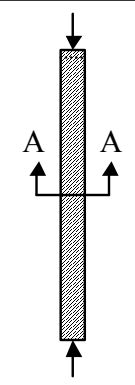

\section{Loading and de formation}

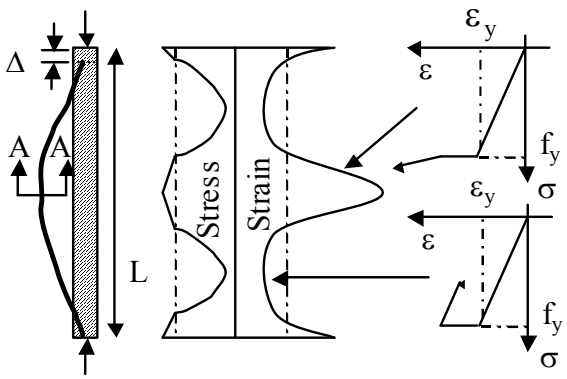

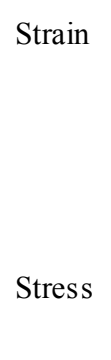

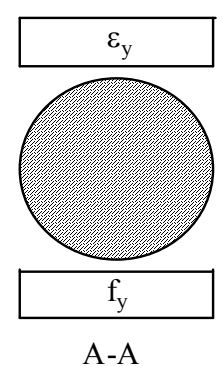

Variation across the section
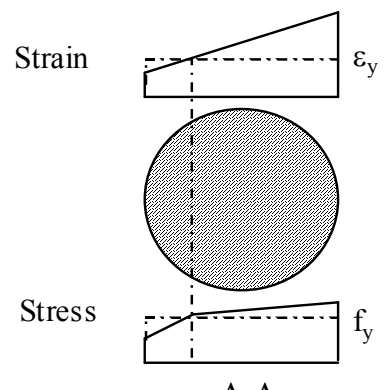

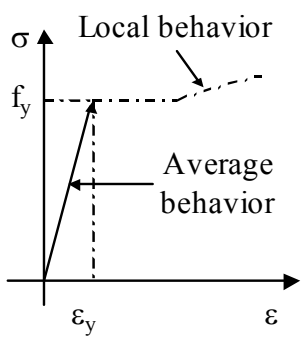

Average and local behavions

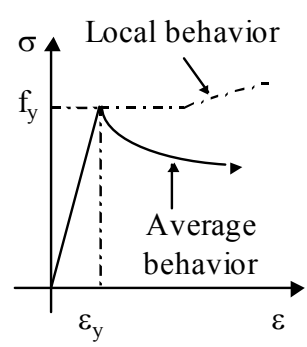

After buckling $\varepsilon=\Delta /$ L: Averaged strain;

$\mathrm{L}$ : Referential length in which average constitutive model is defined

Fig. 2 

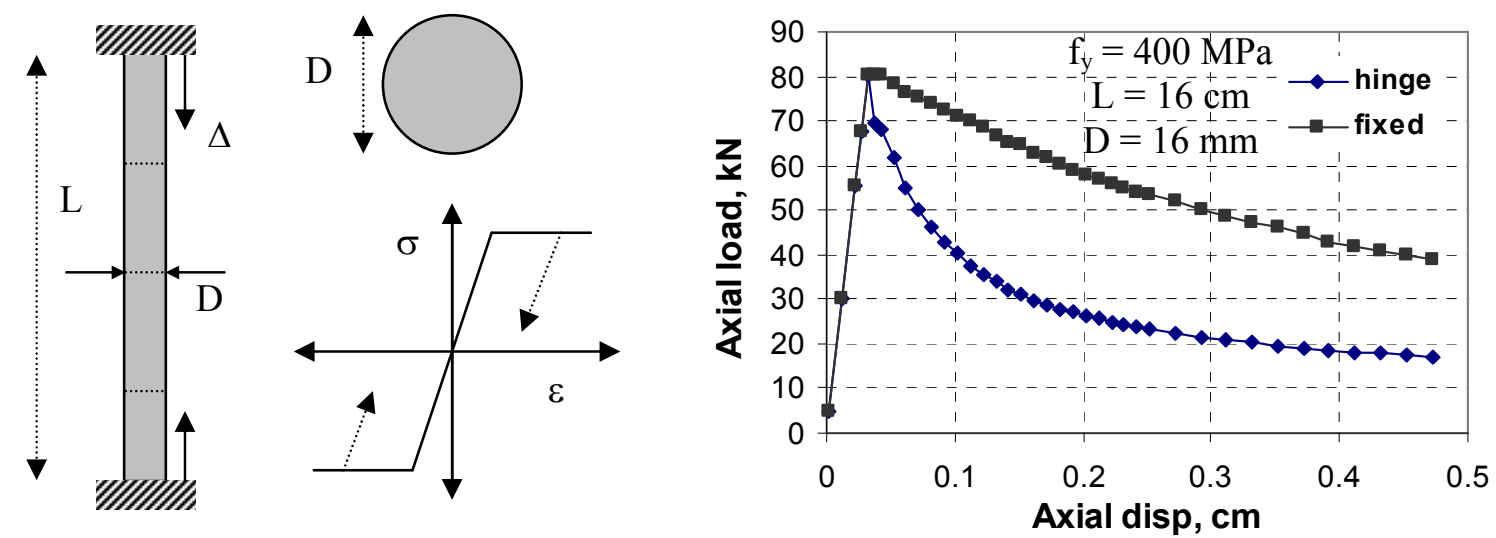

Fig. 3 

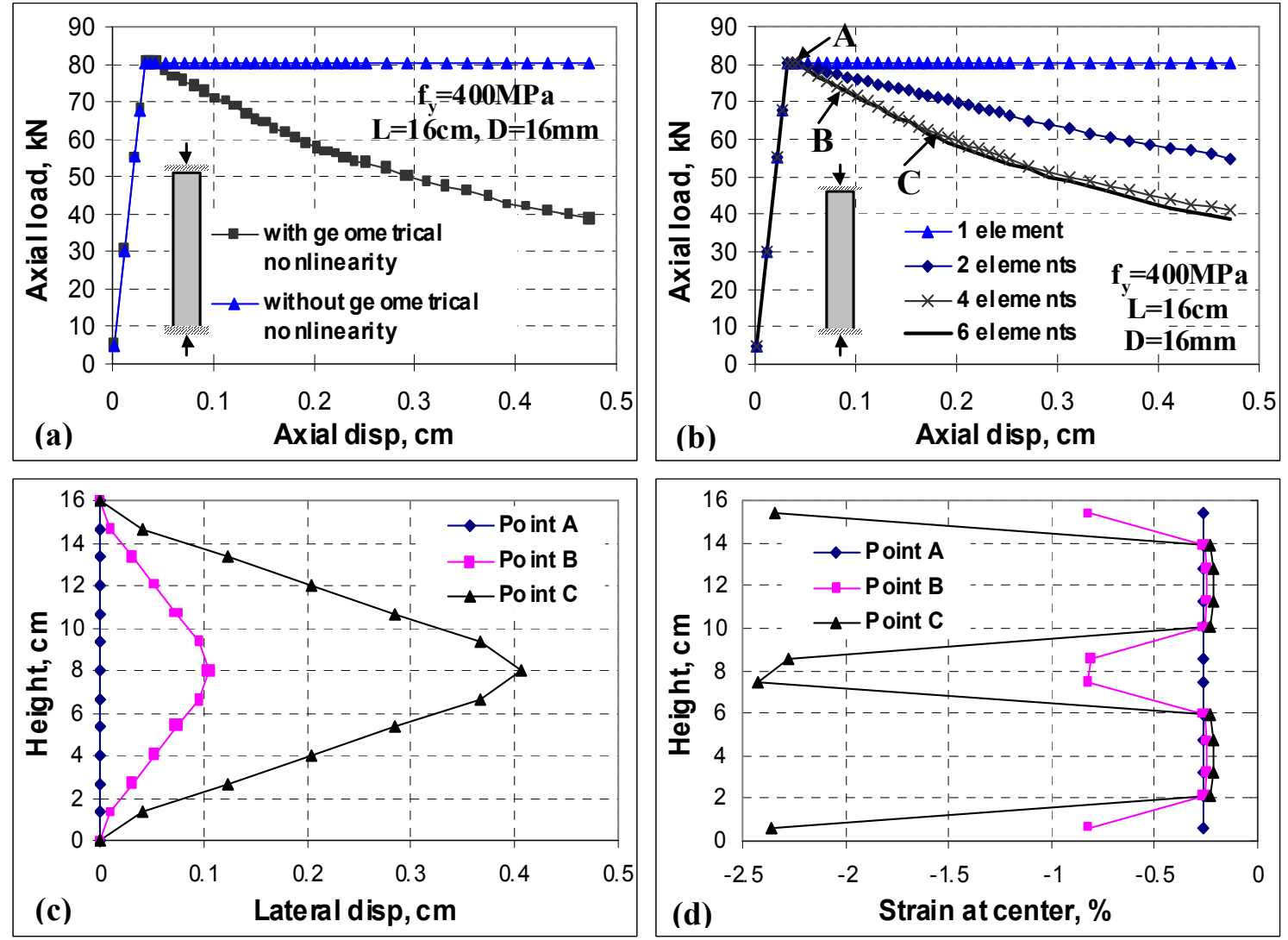

Fig. 4 


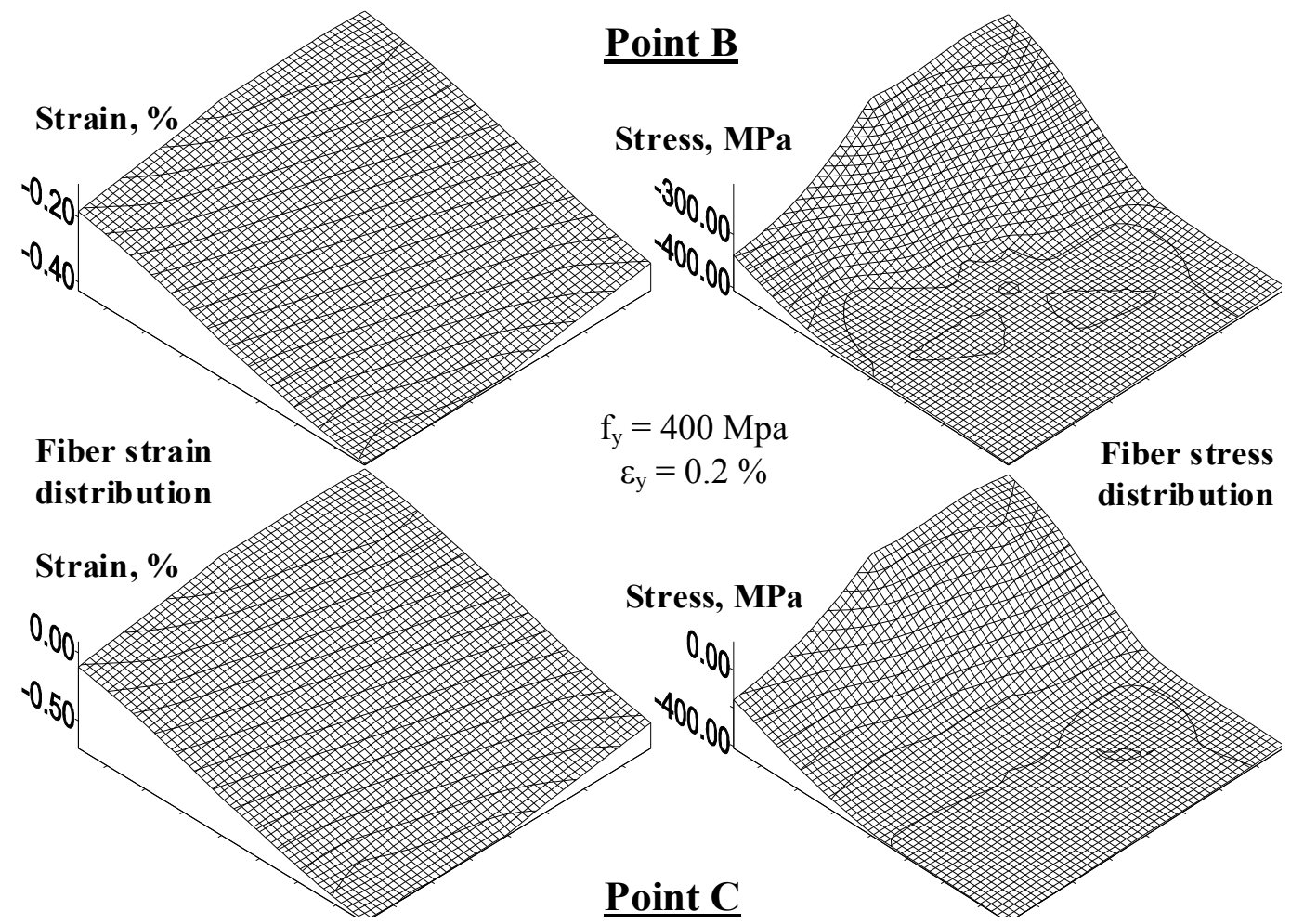

Fig. 5 

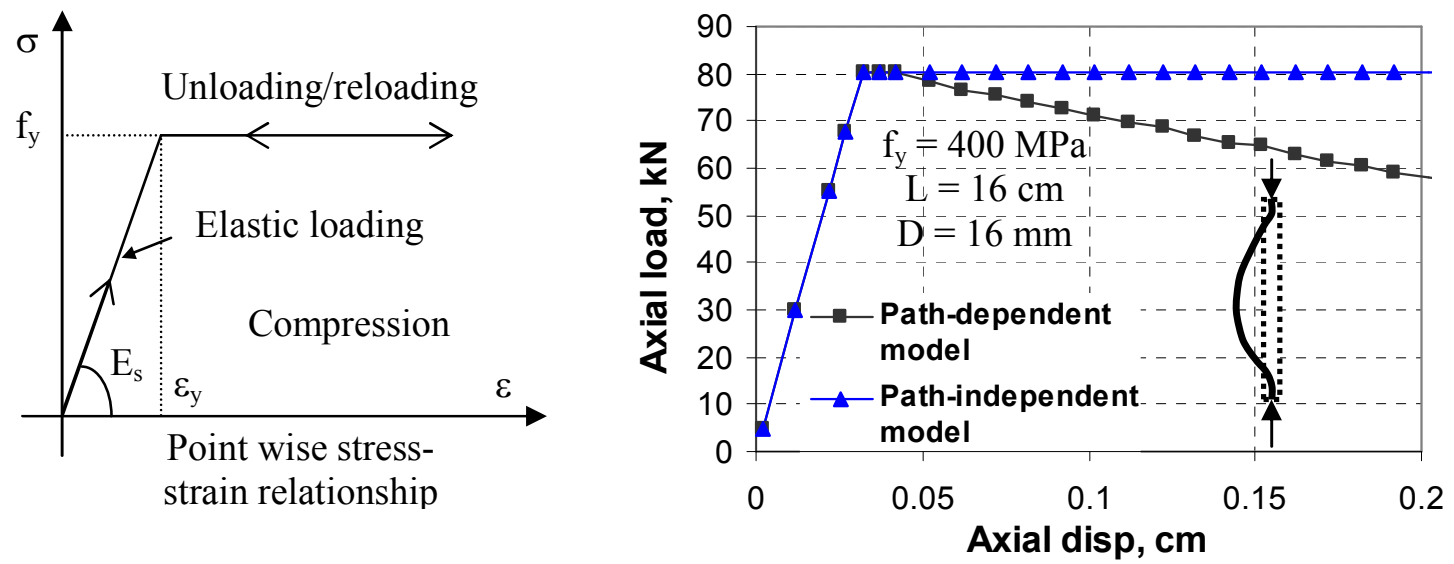

Fig. 6 

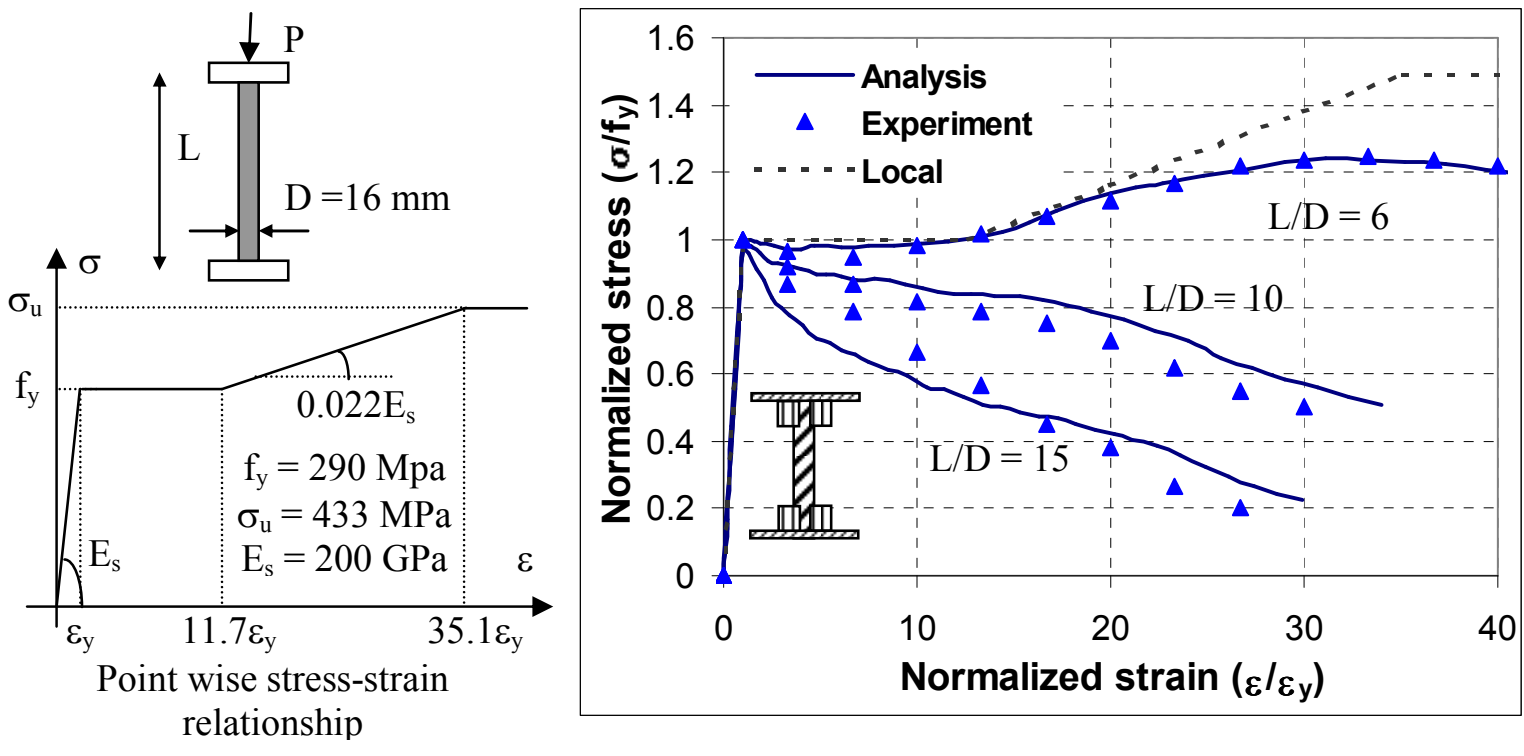

Fig. 7 

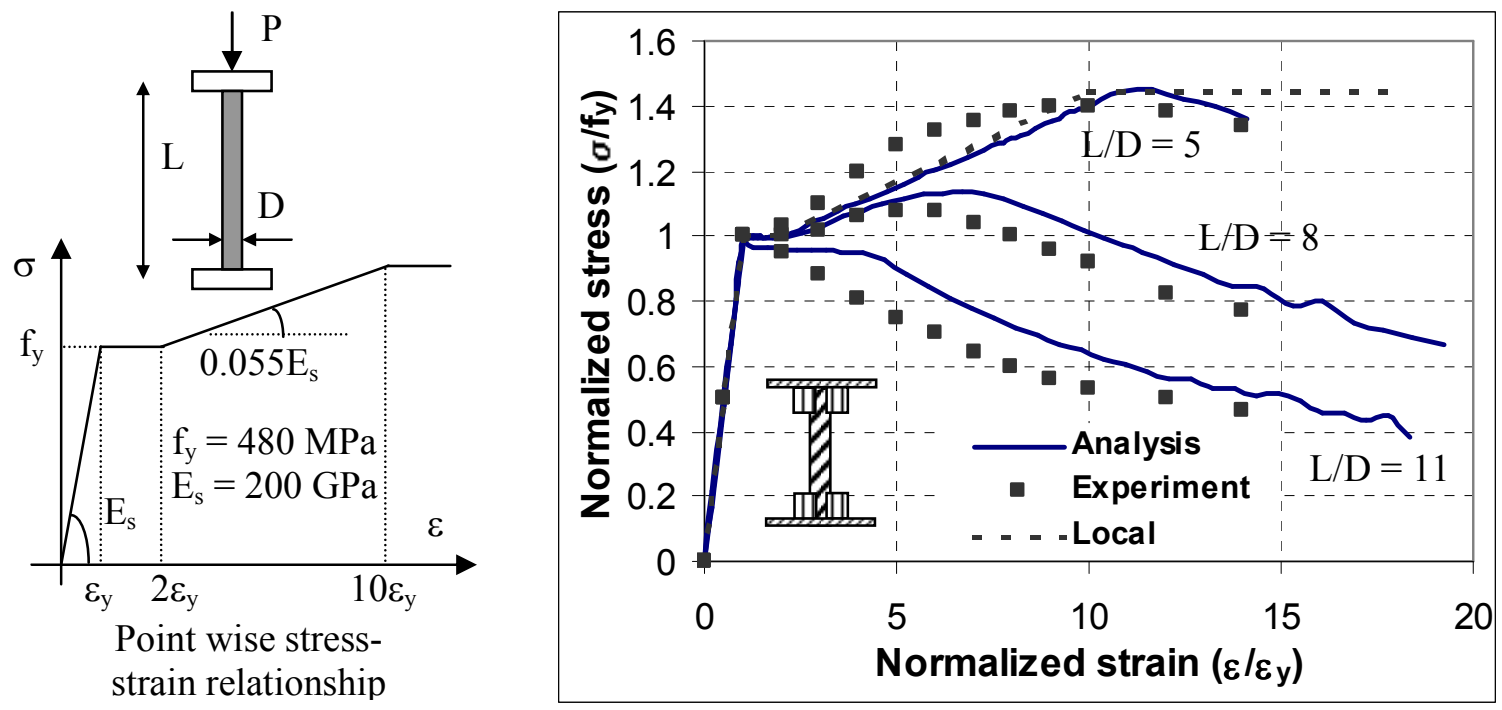

Fig. 8 

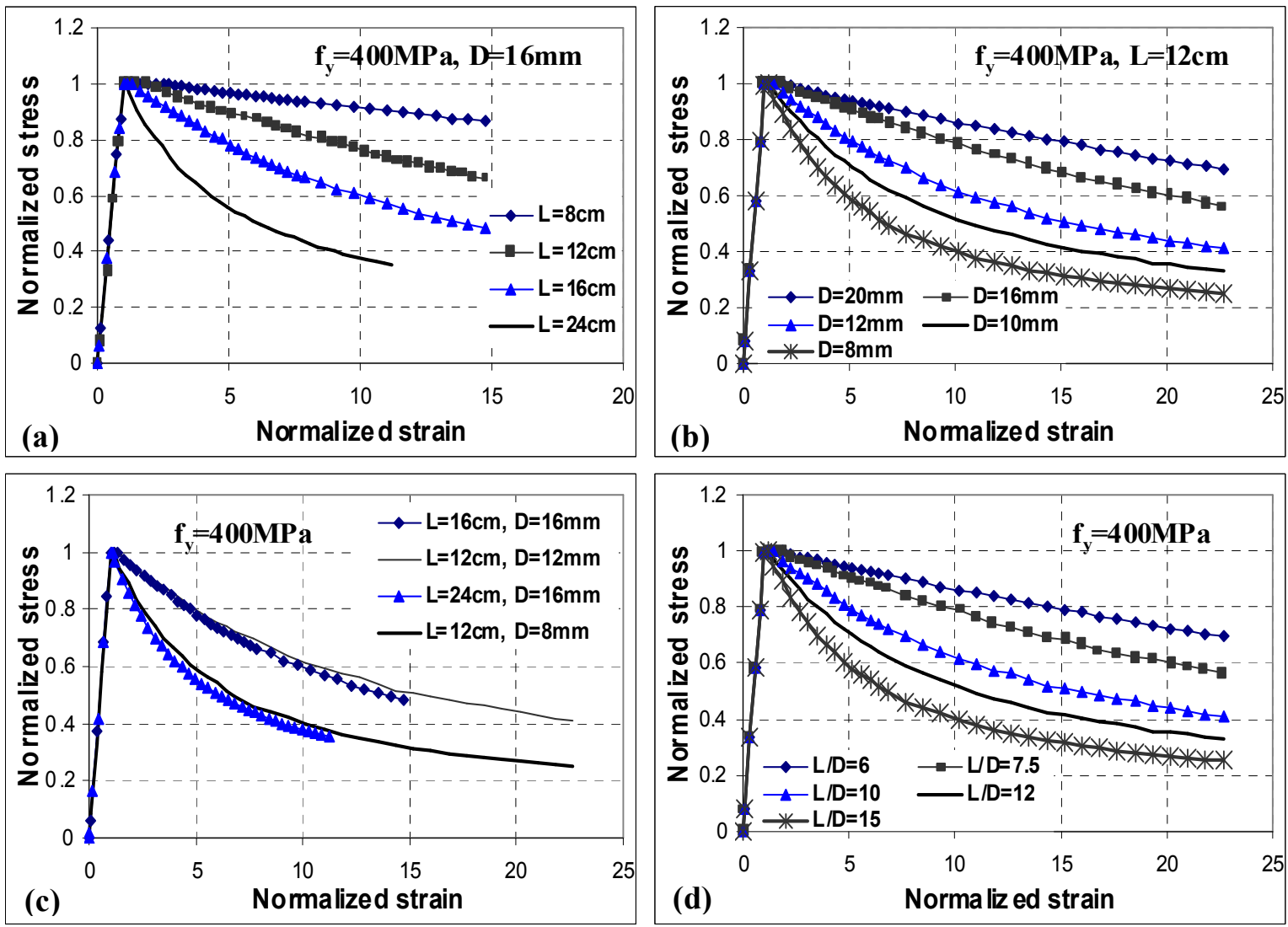

Fig. 9 

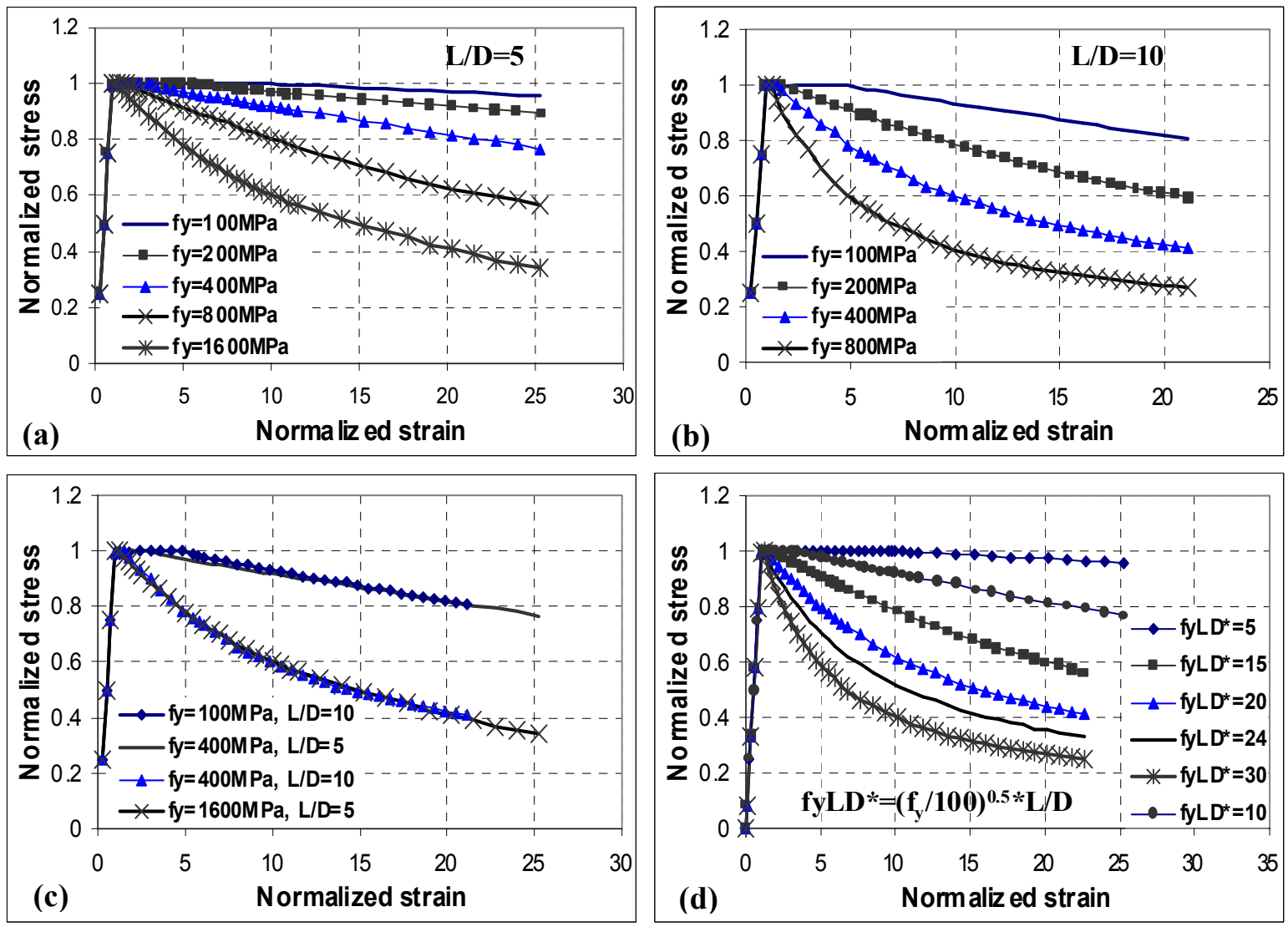

Fig. 10 

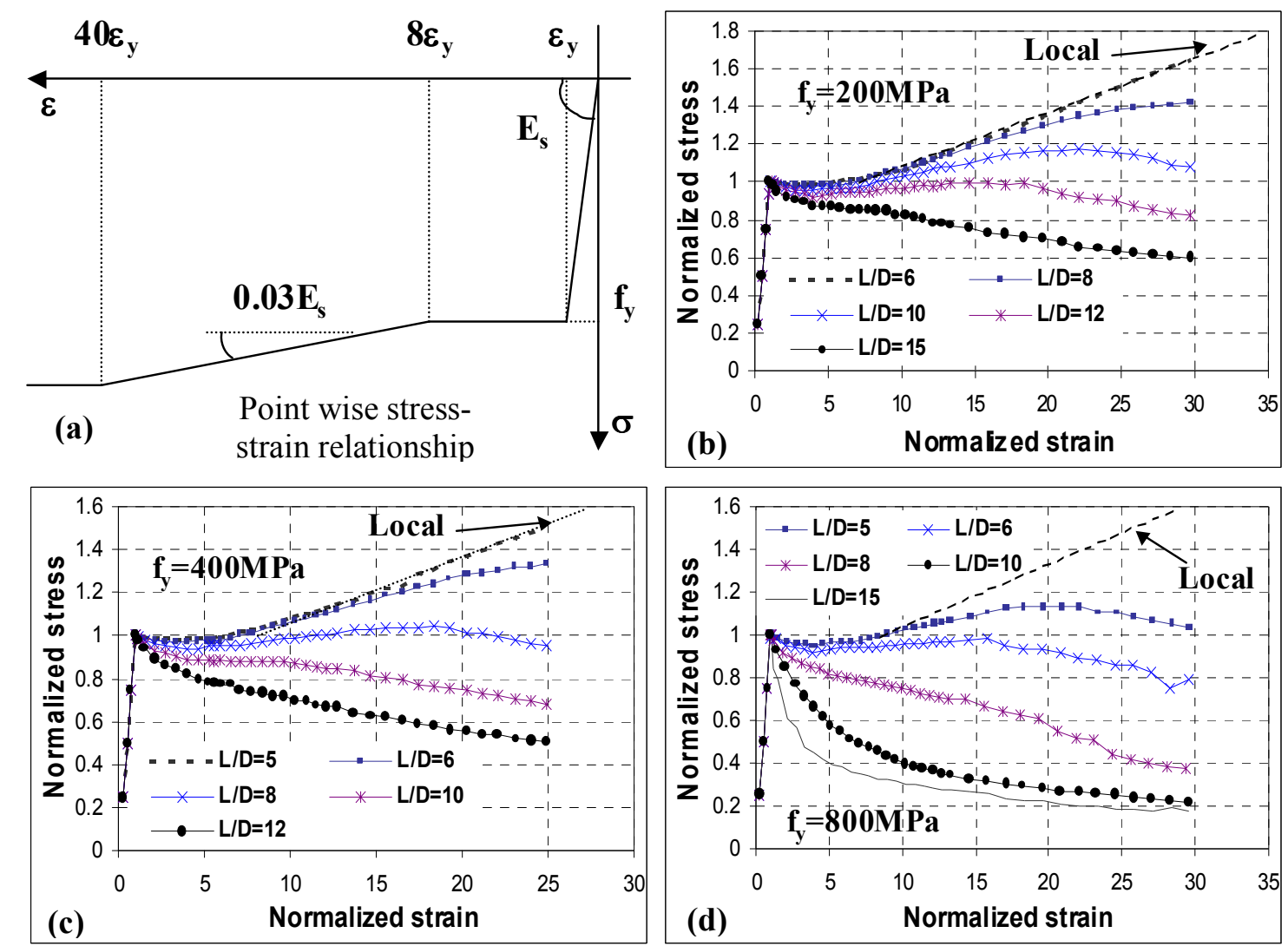

Fig. 11 


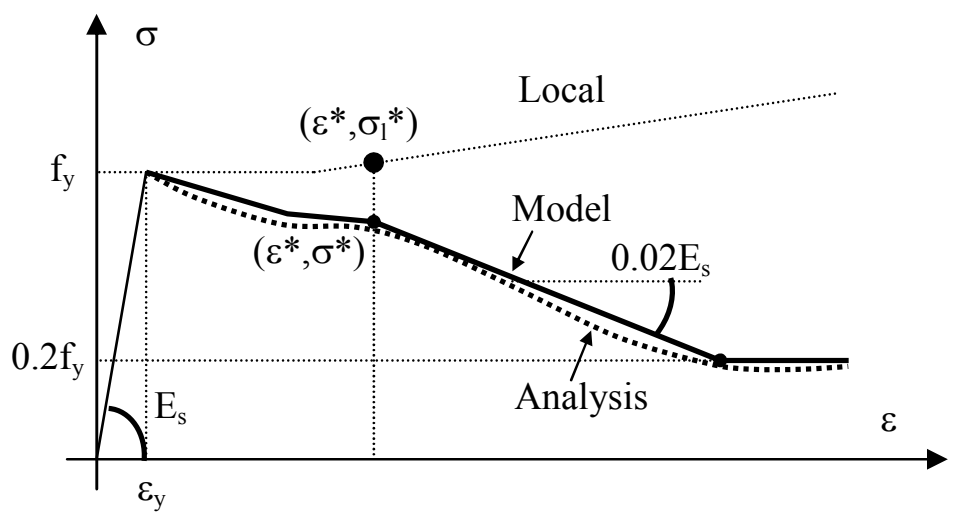

Fig. 12 

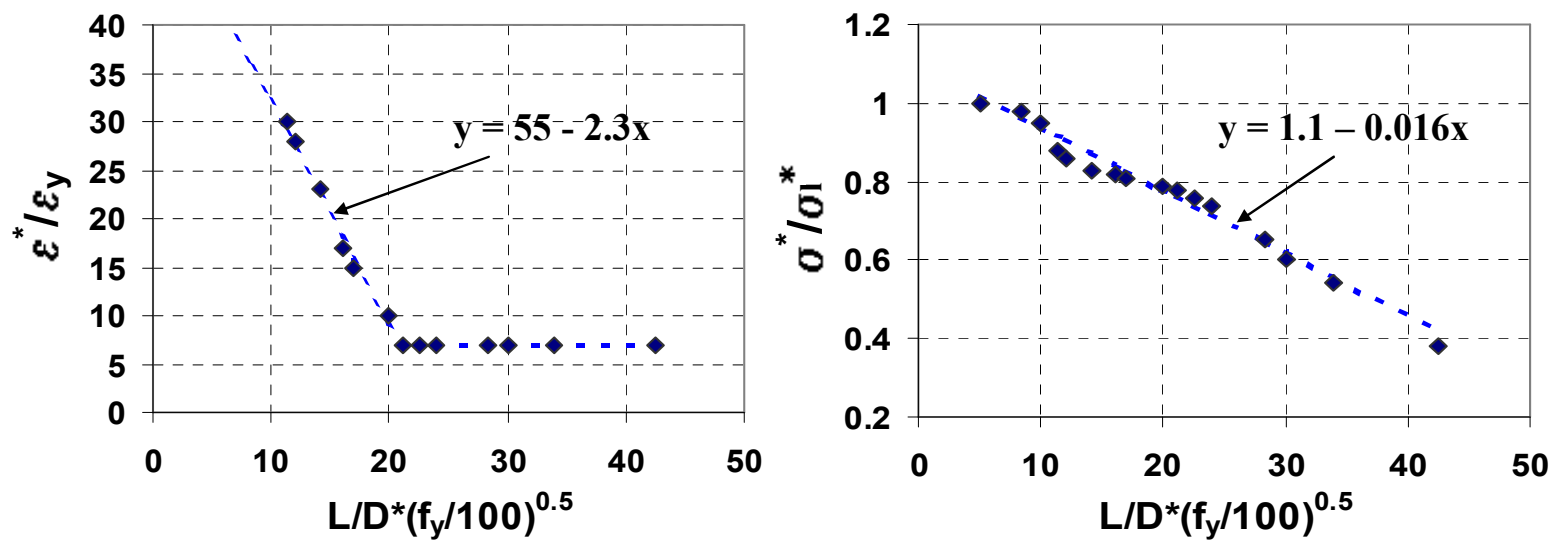

Fig. 13 


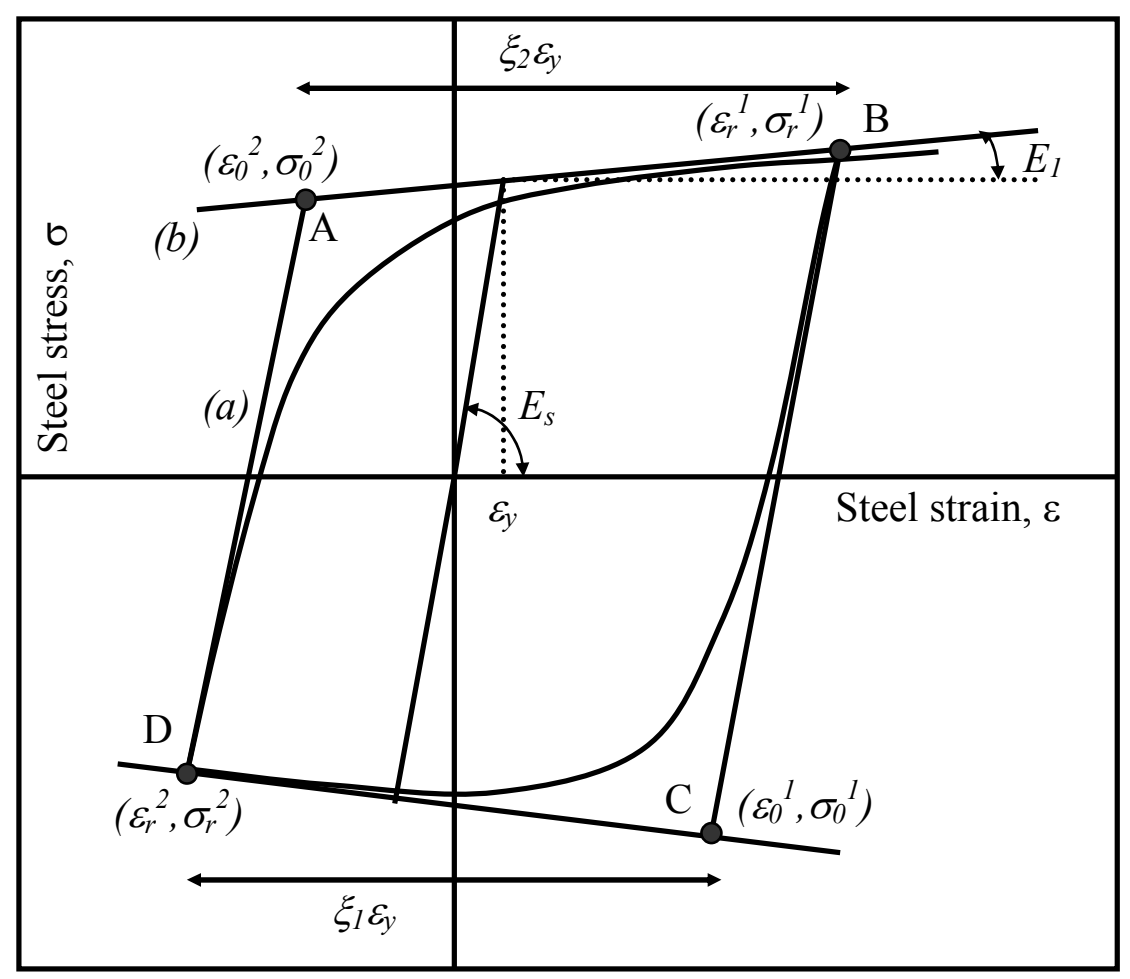

Fig. 14 

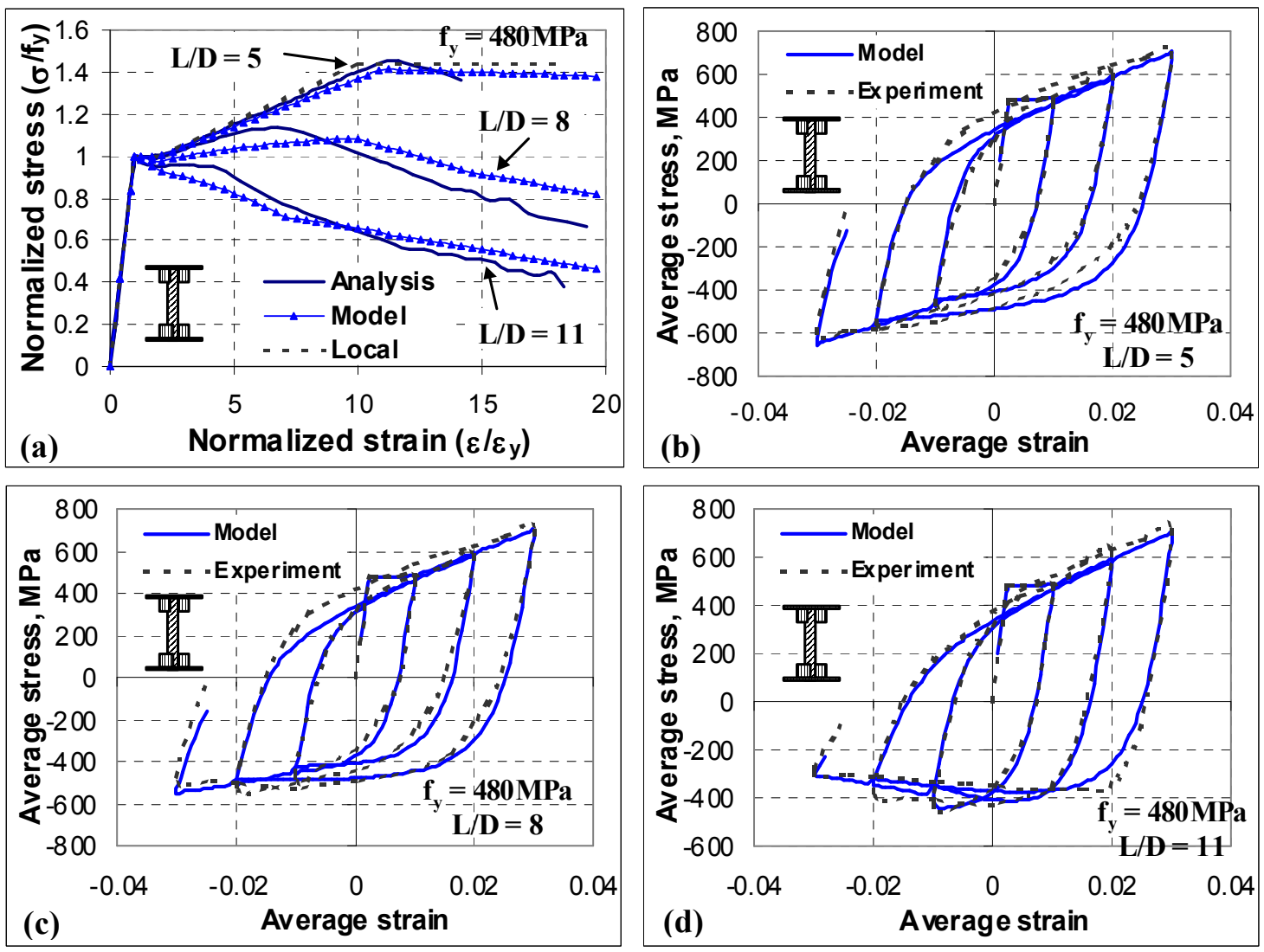

Fig. 15 

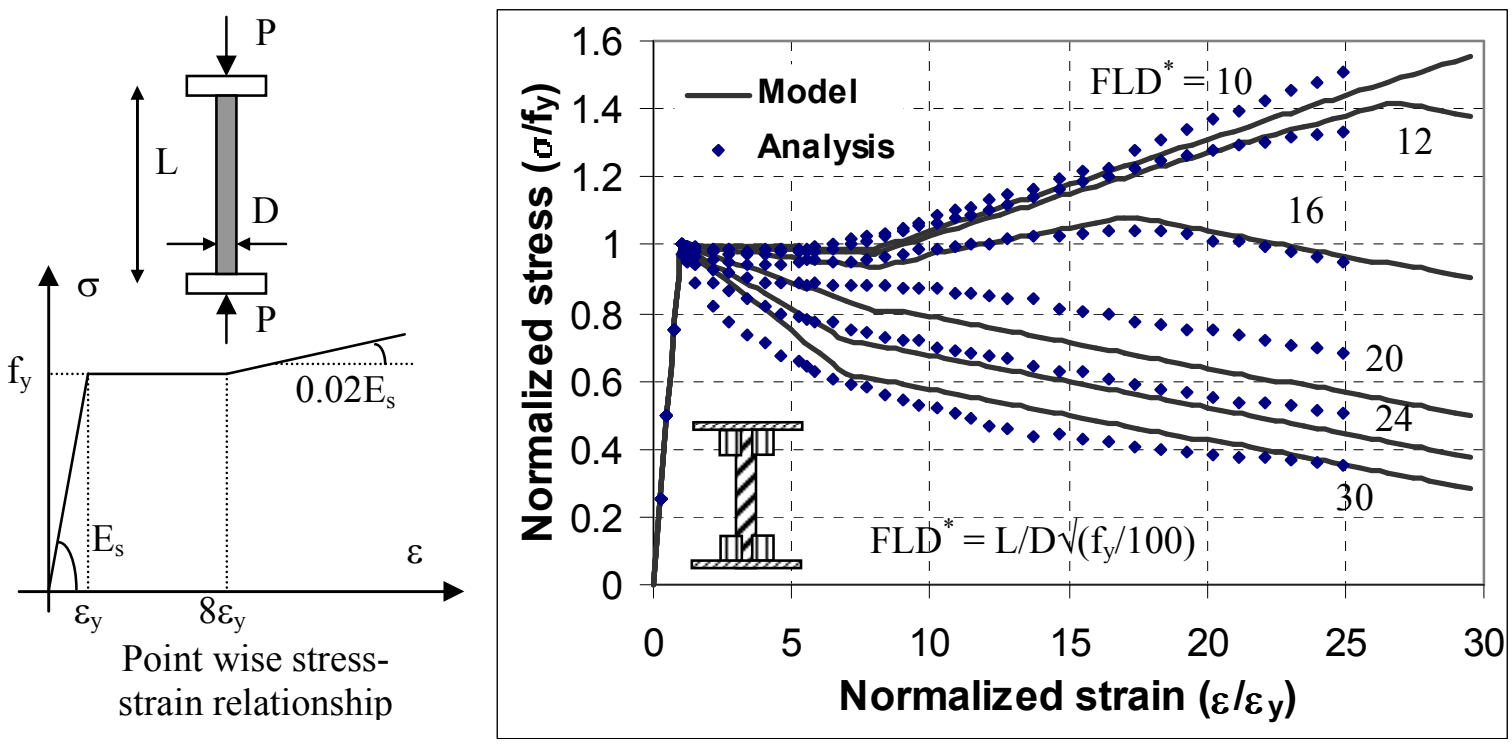

Fig. 16 

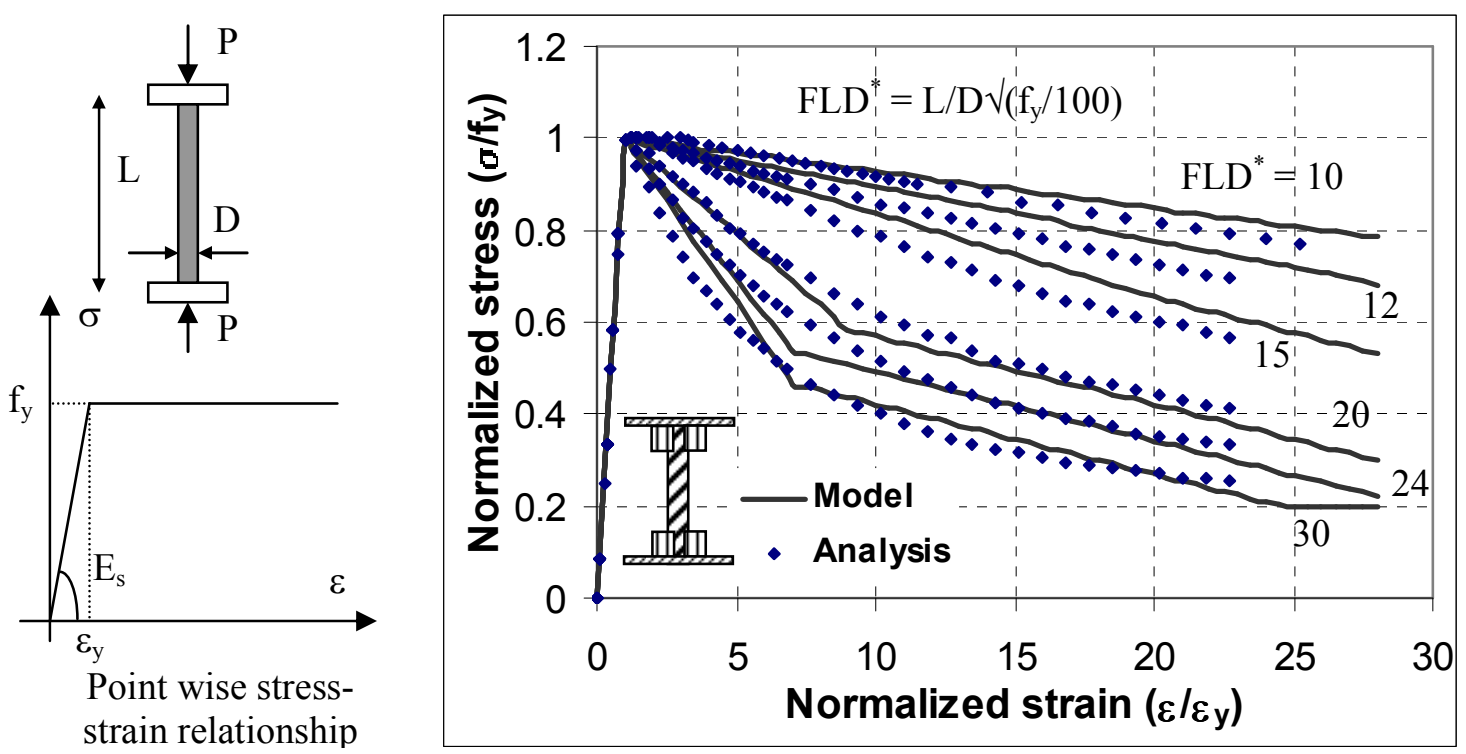

Fig. 17 